\begin{tabular}{|r|l|}
\hline \multicolumn{2}{|c|}{ Statistica Sinica Preprint No: SS-2021-0164 } \\
\hline Title & $\begin{array}{l}\text { Mendelian Randomization Test of Causal Effect Using } \\
\text { High-Dimensional Summary Data }\end{array}$ \\
\hline Manuscript ID & SS-2021-0164 \\
\hline URL & http://www.stat.sinica.edu.tw/statistica/ \\
\hline DOI & $10.5705 /$ ss.202021.0164 \\
\hline Complete List of Authors & $\begin{array}{l}\text { Lu Deng } \\
\text { William Wheeler and } \\
\\
\text { Kai Yu }\end{array}$ \\
\hline Corresponding Author & Kai Yu \\
\hline E-mail & yuka@mail.nih.gov \\
\hline Notice: Accepted version subject to English editing. \\
\hline
\end{tabular}


Statistica Sinica

\title{
MENDELIAN RANDOMIZATION TEST OF CAUSAL EFFECT USING HIGH-DIMENSIONAL SUMMARY DATA
}

\author{
Lu Deng ${ }^{1}$, William Wheeler ${ }^{2}$ and Kai $\mathrm{Yu}^{3 *}$ \\ ${ }^{1}$ School of Statistics and Data Science, Nankai University, Tianjin, P. R. China \\ ${ }^{2}$ Information Management Services, Silver Spring, MD, U.S.A. \\ ${ }^{3}$ Division of Cancer Epidemiology and Genetics, National Cancer Institute, Bethesda, MD, U.S.A. \\ *corresponding author yuka@mail.nih.gov
}

\begin{abstract}
Mendelian randomization (MR) uses genetic variants as instrumental variables (IVs) to assess the causal effect of a risk factor on an outcome in the presence of unmeasured confounding. There is a growing interest to conduct MR analyses using summary statistics on each IV's association with the risk factor and with the outcome, which are generated from large-scale genome-wide association studies (GWAS). Most existing approaches use summary data on a set of IVs that have been established to be associated with the risk factor. They often have limited power due to the fact that the set of identified IVs jointly only explain a small proportion of variation in the measure of the risk factor. We propose a new MR testing procedure that takes full advantage of summary data on tens of thousands of genetic variants studied by GWAS. The testing statistic is the maximum of a sequence of modified K-statistics (Kleibergen, 2002) defined by a range of thresholds. Compared to existing approaches, this new test gains its power by collecting signals from many undetected IVs throughout the genome and is robust to both balanced and unbalanced pleiotropy. We investigate the theoretic properties of the proposed procedure and demonstrate its advantage over existing ones through simulation studies and a real example.
\end{abstract}


Key words and phrases: Genome-wide association studies, Instrumental variables, Mendelian randomization, Pleiotropic effect, Summary statistics.

\section{Introduction}

Mendelian randomization (MR) analysis uses genetic variants as instrumental variables (IVs) to estimate the causal effect of a risk factor on an outcome based on observational studies (Lawlor et al., 2008; Smith and Ebrahim, 2003). It is becoming a very effective tool to study the causal relationship between a risk exposure and a disease outcome, as many robust findings on genetic basis underlying various common traits have been accumulated through large-scale genome-wide association studies (GWAS) over the past decade.

Most MR analyses are conducted under the two-sample setting by combining summary data from two separate GWAS, with one study providing summary statistics on the association between each IV (typically, a single nucleotide polymorphism called SNP) and the risk factor, and the other giving summary statistics on IV and outcome association Burgess et al. 2015; Hemani et al., 2018). Since the two-sample MR analysis only uses SNP association summary statistics, its input data is easy to obtain and assemble. With more summary data becoming publicly available, two-sample MR studies are carried out routinely in the analysis of GWAS.

A typical MR analysis relies on the identification of a set of IVs that are expected to meet the following three conditions (Didelez and Sheehan, 2007). 1. Relevance: each IV is associated with the risk factor $X$. 2. Exclusion Restriction: each IV only affects the outcome $Y$ through its influence on $X$. 3. Effective Random Assignment: all IVs are jointly independent of any unmeasured confounder that correlates with both $X$ and $Y$. Most MR procedures satisfy the Relevance Assumption by using SNPs that either have been established by other studies to be associated with the risk factor, or have demonstrated genome-wide significant association with the risk factor (e.g., $p$-value less than $5 \times 10^{-8}$ ) in the same study that provides the summary data (Bowden et al., 2016; Bowden et al., 2017; Burgess et al. 
2016; Burgess et al., 2013; Burgess et al., 2020; Hartwig et al., 2017; Qi and Chatterjee, 2019).

Quite a few approaches have been proposed for using invalid instruments that violate either the Exclusive Restriction or Effective Random Assignment assumption. In the setting of MR analysis, those invalid IVs can be SNPs with pleiotropic effects, i.e., SNPs that can affect both the risk factor and the outcome. Some of those approaches require the use of individual-level raw data (e.g., Guo et al., 2018; Kang et al., 2016; Kang et al., 2020; Tchetgen et al., 2017; Windmeijer et al., 2018). Most recently developed MR procedures focus on using summary data generated from GWAS as input and allow some of the chosen IVs (SNPs) to have pleiotropic effects (e.g., Bowden et al., 2015; Bowden et al., 2016; Burgess et al., 2020; Hartwig et al., 2017; Mprrison et al., 2020; Qi and Chatterjee, 2019; Xue et al., 2021). They usually choose genome-wide significant SNPs as IVs to satisfy the Relevance condition, and require additional assumptions, such as plurality validity, to ensure the identifiability of model parameters Guo et al., 2018; Xue et al., 2021).

The strategy of using only genome-wide significant SNPs as IVs works fine for risk factors that have been studied by large scale GWAS, such as BMI and blood pressure (Evangelou et al., 2018; Yengo et al., 2018), as these studies have adequate power to identify hundreds of genome-wide significant SNPs to be selected as IVs. But for a vast majority of other risk factors, the number of qualified SNPs are well below 100. MR analyses with those risk factors would have limited power as the set of chosen IVs might only explain a small proportion of the total variation of the risk factor (Deng et al., 2020).

The use of selected SNPs as IVs has another potential limitation. When the same GWAS is used for the selection of IVs and for providing summary statistics, there would be the winner's curse effect on the summary data (Siegmund, 2002; Yu et al., 2007). For SNPs barely passing the selection threshold, their levels of association with the risk factor tend to be over- 
estimated. Using biased summary statistics in a MR study can lead to erroneous conclusions (Zhao et al., 2020). Zhao et al. (2019) proposed a three-sample genome-wide design that utilizes summary data from three independent GWAS, with one GWAS used for the selection of IVs showing evidence of association with the risk factor, and the other two GWAS for the two-sample MR analysis. Since the GWAS used for selecting IVs is not used in the MR analysis, this three-sample design avoids the winner's curse effect and allows the use of many IVs (around 1,000) for more effective evaluation of the causal effect. However, it is not clear how to select an appropriate set of IVs to achieve the optimal performance.

We propose a two-sample MR procedure to test whether there exists a causal effect of the risk factor on the outcome. The new approach uses summary data on all independent SNPs from GWAS, instead of just a few that are genome-wide significant. The test statistic is the maximum of a sequence of modified K-statistics (Kleibergen, 2002) defined by a range of thresholds. Each K-statistic in the sequence is calculated with summary data on selected SNPs whose correlations with the risk factor are above a given threshold. We use this maximal thresholding statistic to optimize the power for detecting the causal effect manifested through an unspecified set of SNPs. A similar idea was used to identify sparse signals in simultaneous tests of a large number of unrelated hypotheses (Donoho and Jin, 2004, Zhong et al., 2013). The proposed approach gains its power by collecting signals from many weak IVs, but it focuses on testing instead of estimating the causal effect. It is a two-sample MR testing procedure and does not require an additional GWAS for the selection of IVs. The new approach uses a data-driven threshold to identify the optimal set of SNPs for the test of the causal effect.

\section{Method}

\subsection{Setup and notations}

Let $Y, X$, and $\mathbf{Z}$ represent the outcome, the risk factor, and the vec- 
tor of genotypes on $p$ independent SNPs, respectively. Here we consider all independent SNPs extracted from GWAS, with $p$ possibly larger than 100,000. In the two-sample MR setting, we have summary data on the association between each SNP and the outcome from the GWAS of $Y$, and summary data on the association between each SNP and the risk factor from the GWAS of $X$. We denote the summary data from the outcome GWAS as $\left\{\left(\hat{\beta}_{Y k}, \sigma_{Y k}^{2}\right), k=1, \ldots, p\right\}$, with $\hat{\beta}_{Y k}$ being the estimated regression coefficient on the association between the $k^{\text {th }} \mathrm{SNP}$ and $Y$, and $\sigma_{Y k}$ being the corresponding standard error. We denote the summary data from the risk factor GWAS as $\left\{\left(\hat{\beta}_{X k}, \sigma_{X k}^{2}\right), k=1, \ldots, p\right\}$. Similar to most two-sample MR procedures, we assume the two GWAS are conducted in the same population. If they come from different populations, our proposed method described later still works under the structural invariance assumption, i.e., the underlying models for the risk factor and the outcome remain the same between the two populations (Zhao et al., 2019).

As done in many two-sample MR procedures, we assume $\hat{\beta}_{Y k} \sim N\left(\beta_{Y k}\right.$, $\left.\sigma_{Y k}^{2}\right)$ and $\hat{\beta}_{X k} \sim N\left(\beta_{X k}, \sigma_{X k}^{2}\right)$, with unknown $\beta_{Y k}$ and $\beta_{X k}$ representing $k^{\text {th }}$ SNP's true marginal effect on $Y$ and $X$, respectively. We consider $\beta_{X k}, k=1, \ldots, p$, as i.i.d. random samples from a mixture distribution, i.e., $\beta_{X k} \sim \lambda f_{X}+(1-\lambda) \delta$, with $f_{X}$ being an arbitrary distribution, $\delta$ being the degenerated distribution taking a constant value 0 , and $\lambda$ being the mixture proportion. We let $d_{k}=1$ if $\beta_{X k}$ takes value from $f_{X}, d_{k}=2$ otherwise. Therefore, the $k^{\text {th }} \mathrm{SNP}$ is associated with $X$ if $d_{k}=1$. A SNP can affect $Y$ in several ways simultaneously. It can influence $Y$ directly, through its effect on $X$, or other mediated factors. $\beta_{Y k}$ represents the $k^{\text {th }}$ SNP's true marginal effect on $Y$, summarizing all those effects. We treat $\sigma_{Y k}$ and $\sigma_{X k}$ as known constant values.

Since we consider a set of independent SNPs in the setting of twosample MR study, the $p$ random variables $\hat{\beta}_{Y k}, k=1, \ldots, p$, are mutually independent. This is also true for $\hat{\beta}_{X k}, k=1, \ldots, p$. Zhao et al. (2020) provided further justification. Also, as the summary data comes from two 
independent studies, we can assume that $\left(\hat{\beta}_{Y k}, \hat{\beta}_{Y k^{\prime}}, \hat{\beta}_{X k}, \hat{\beta}_{X k^{\prime}}\right)$ are conditionally independent given the true marginal effects. We call data satisfying those conditions the two-sample independent summary data in the following discussions.

\subsection{Method under the genome-wide InSIDE Assumption}

When a large set of SNPs are considered, it is inevitable that some of them can have pleiotropic effects. Let $\alpha_{k}$ be the $k^{t h}$ SNP's pleiotropic effect on $Y$, apart from the one mediated through $X$. Under the standard InSIDE (Instrument Strength Independent of Direct Effect) assumption (Bowden et al. 2015), $\alpha_{k}$ and $\beta_{X k}$ are independent. The InSIDE assumption is typically made on the set of SNPs that are associated with the risk factor. We extend this assumption to SNPs throughout the genome and called it the genome-wide InSIDE assumption, which can be specified as the following.

Genome-wide InSIDE Assumption. For $k=1, \ldots, p, \beta_{Y k}=$ $\theta \beta_{X k}+\alpha_{k}$, with $\alpha_{k} \perp \beta_{X k}$, and $\alpha_{k}$ being identical and independent random variables.

In this assumption, we regard $\alpha_{k}, k=1, \ldots, p$, as i.i.d. random effects that are independent of $\beta_{X k}$ and have the same distribution as $\alpha$, with $E(\alpha)=\mu$ and $\operatorname{Var}(\alpha)=\omega^{2}$. The distribution of $\alpha$ is unspecified. It can be a mixture distribution, similar to that of $\beta_{X k}$. $\theta$ is a constant value representing the causal effect of the risk factor. The null hypothesis of the MR test is $H_{0}: \theta=0$.

We first present the test statistic assuming that the mean $(\mu)$ and variance $\left(\omega^{2}\right)$ of $\alpha$ are known. Then, we provide estimates of $\mu$ and $\omega^{2}$, and show the property of the test statistic with the two estimates being plugged in. We consider the following threshold K-statistic (Kleibergen, 2002; Wang and Kang, 2019),

$$
Q(s)=\sum_{k=1}^{p}\left\{\frac{\left(\hat{\beta}_{Y k}-\mu\right)}{\sqrt{\omega^{2}+\sigma_{Y k}^{2}}} \frac{\hat{\beta}_{X k}}{\sigma_{X k}}\right\} I\left(\frac{\hat{\beta}_{X k}^{2}}{\sigma_{X k}^{2}} \geq 2 s \log p\right),
$$


where $I(\cdot)$ is an indicator function, and $s$ is a chosen threshold parameter that takes a value within $[0,1)$. The intuition for using a threshold on the K-statistic is to give more weight to SNPs that are likely to be associated with $X$. We consider the threshold at the scale of $2 \log p$ because of the large deviations result (Petrov, 1995), which implies that if $\beta_{X k}=0$ for all $1 \leq k \leq p$, then $\operatorname{Pr}\left(\max _{1 \leq k \leq p} \hat{\beta}_{X k}^{2} / \sigma_{X k}^{2} \leq 2 \log p\right) \rightarrow 1$ as $p \rightarrow \infty$. We require $s$ to be less than one to ensure a sufficient number of SNPs (e.g., larger than 20) passing that threshold, as we require $Q(s)$ to be asymptotic normal in the proof (see Supplementary Material S1).

One distinctive feature of $Q(s)$ defined in (1) is that the distribution of $\hat{\beta}_{X k}^{2} / \sigma_{X k}^{2}$ inside the indictor function is unknown under the null, as we do not know which SNP is associated with the outcome. This is different from those threshold statistics considered by Zhong et al. (2013). By assuming $\mu$ and $\omega^{2}$ are known, based on the genome-wide InSIDE assumption we can calculate the mean of $Q(s)$ as

$$
\begin{aligned}
& E_{\theta}\{Q(s)\}=\sum_{k=1}^{p} E_{\theta}\left\{\frac{\left(\hat{\beta}_{Y k}-\mu\right)}{\sqrt{\omega^{2}+\sigma_{Y k}^{2}}}\right\} E_{\theta}\left\{\frac{\hat{\beta}_{X k}}{\sigma_{X k}} I\left(\frac{\hat{\beta}_{X k}^{2}}{\sigma_{X k}^{2}} \geq 2 s \log p\right)\right\} \\
= & \sum_{k=1}^{p} \frac{\theta \beta_{X k}}{\sqrt{\omega^{2}+\sigma_{Y k}^{2}}}\left[\frac{\beta_{X k}}{\sigma_{X k}}\left\{\bar{\Phi}\left(\sqrt{2 s \log p}+\frac{\beta_{X k}}{\sigma_{X k}}\right)+\bar{\Phi}\left(\sqrt{2 s \log p}-\frac{\beta_{X k}}{\sigma_{X k}}\right)\right\}\right. \\
& \left.+\phi\left(\sqrt{2 s \log p}-\frac{\beta_{X k}}{\sigma_{X k}}\right)-\phi\left(\sqrt{2 s \log p}+\frac{\beta_{X k}}{\sigma_{X k}}\right)\right] \equiv \theta \sum_{k=1}^{p} h\left(\beta_{X k}\right),
\end{aligned}
$$

where $\phi(\cdot), \bar{\Phi}(\cdot)$ are the density function and survival function of the standard normal distribution, respectively. Here and throughout this paper the expectation is calculated over the summary data, as well as the unobserved random effect $\alpha_{k}$, conditioning on $\beta_{X k}$. Since $h\left(\beta_{X k}\right)=h\left(-\beta_{X k}\right)$, and $h\left(\beta_{X k}\right) \geq 0$ for $\beta_{X k} \geq 0, E_{\theta}\left\{Q_{p}(s)\right\}$ has the same sign as $\theta$. It can be noted that if all $\beta_{X k}=0$, then $E_{\theta}\left\{Q_{p}(s)\right\}=0$ regardless of $\theta=0$ or not. Therefore, the proposed test has no power when all SNPs are not associated with $X$.

Under the null, we have $E_{\theta=0}\{Q(s)\}=0$. The variance of $Q(s)$ under 
the null can be written as

$$
V^{2}(s) \equiv \operatorname{Var}_{\theta=0}\{Q(s)\}=\sum_{k=1}^{p} E_{\theta=0}\left\{\frac{\hat{\beta}_{X k}^{2}}{\sigma_{X k}^{2}} I\left(\frac{\hat{\beta}_{X k}^{2}}{\sigma_{X k}^{2}} \geq 2 s \log p\right)\right\} .
$$

In practice, we can estimate $V^{2}(s)$ as

$$
\hat{V}^{2}(s)=\sum_{k=1}^{p} \frac{\hat{\beta}_{X k}^{2}}{\sigma_{X k}^{2}} I\left(\frac{\hat{\beta}_{X k}^{2}}{\sigma_{X k}^{2}} \geq 2 s \log p\right) .
$$

The following result establishes the asymptotic normality of $Q_{p}(s)$ with its proof given in Supplementary Material S1.

Theorem 1. Given two-sample independent summary data $\left(\hat{\beta}_{Y k}, \sigma_{Y k}^{2}, \hat{\beta}_{X k}\right.$, $\left.\sigma_{X k}^{2}\right), k=1, \ldots, p$, under the genome-wide InSIDE assumption, we have for any fixed $s \in(0,1)$, as $p \rightarrow \infty, V^{-1}(s) Q(s) \stackrel{D}{\rightarrow} N(0,1)$ and $\hat{V}^{-1}(s) Q(s) \stackrel{D}{\rightarrow}$ $N(0,1)$ under $H_{0}: \theta=0$.

We can use this result to construct the standardized test statistic $\hat{V}^{-1}(s)$ $Q(s)$ to test $H_{0}: \theta=0$. It is interesting to notice that the calculation of $\hat{V}^{2}(s)$ in (4) does not need $\hat{\beta}_{Y k}$ and $\sigma_{Y k}^{2}$. The above test statistic (1) relies on the choice of a threshold $s$. A more effective approach is to consider all possible thresholds within a given range, then choose the one that leads to an optimal test adaptive to the signal-to-noise ratio in the data. Therefore, we propose the following maximal thresholding statistic, called two-sided MaxK test statistic,

$$
T=\max _{s \in \mathcal{S}} \hat{V}^{-1}|Q(s)|,
$$

where $\mathcal{S}=\left[s_{a}, s_{b}\right]$. To search for the range as wide as possible, we can let $s_{a}=0$, and $s_{b}$ be a value close to 1 , such as $s_{b}=0.98$. We will use this range in the simulation study and the real data application.

Since both $Q(s)$ and $\hat{V}(s)$ are step functions of $s$, we can obtain $T$ given by (5) exactly by checking $\hat{V}^{-1}(s)|Q(s)|$ at a finite number (at most $p$ ) of values for $s$. We derive the asymptotic distribution of $T$ by showing that $\hat{V}^{-1}(s) Q(s)$ follows a Gaussian process and establish the asymptotic 
distribution of $T$ at the tail end (see Supplementary Material S2 for the proof).

Theorem 2. Under conditions given in Theorem 1, we have under $H_{0}$,

$$
\lim _{x \rightarrow+\infty} \frac{1}{x \phi(x)} \operatorname{Pr}(T>x)-2 \tau=0,
$$

where $\tau=2^{-1} \log \left\{\hat{V}^{2}\left(s_{a}\right) / \hat{V}^{2}\left(s_{b}\right)\right\}$.

This result provides an approximation formula to calculate the $p$-value for the two-sided MaxK test. When $T$ is relatively large, we can calculate its $p$-value as $2 T \phi(T) \tau$. Through numeric experiments, we find this formula works very well for approximating relatively small $p$-value (e.g., less than $0.1)$.

According to (2), $E_{\theta}\{Q(s)\}$ has the same sign as $\theta$. Thus, our procedure can be easily extended to test the direction of the causal effect. For example, to target for the one-sided alternative hypothesis $\theta>0$, we can modify the two-sided MaxK statistic as $T_{1}=\max _{s \in \mathcal{S}} \hat{V}^{-1}(s) Q(s)$. Its $p$-value can be calculated as $\left|T_{1}\right| \phi\left(\mid T_{1}\right) \tau$. Similarly, for the alternative $\theta<0$, the one-sided test and its $p$-value can be $T_{2}=\max _{s \in \mathcal{S}}\left\{-\hat{V}^{-1}(s) Q(s)\right\}$ and $\left|T_{2}\right| \phi\left(\mid T_{2}\right) \tau$, respectively.

So far, we assume the mean $(\mu)$ and the variance $\left(\omega^{2}\right)$ of the random effect are known. In practice, we can estimate them using summary data on a set of SNPs that are not associated with $X$. Define $z_{X k}=\hat{\beta}_{X k} / \sigma_{X k}$ and let $\Omega=\left\{k:\left|z_{X k}\right|<z^{*}\right\}$ be the set of SNPs whose $z$-scores for their association with $X$ are below certain threshold $z^{*}$ (e.g., $z^{*}=1.28$, corresponding to a SNP and $X$ association $p$-value of 0.20$)$. Given the fact that most of GWAS SNPs are not associated with $X$, it is reasonable to claim that we have $\beta_{X k}=0$ for $k \in \Omega$. Together with the genome-wide InSIDE assumption, we can estimate $\mu$ and $\omega^{2}$ as

$$
\hat{\mu}=\frac{1}{|\Omega|} \sum_{k \in \Omega} \hat{\beta}_{Y k},
$$




$$
\hat{\omega}^{2}=\frac{1}{|\Omega|} \sum_{k \in \Omega}\left\{\left(\hat{\beta}_{Y k}-\hat{\mu}\right)^{2}-\sigma_{Y k}^{2}\right\},
$$

where $|\Omega|$ is the size of $\Omega$. These two estimates are consistent with any $\theta$ as long as $\beta_{X k}=0$ for $k \in \Omega$.

We can replace $\mu$ and $\omega^{2}$ with their estimates in the calculation of $Q(s)$ and define it as $\hat{Q}(s)$. In application, we can conduct the MaxK test with the following test statistic,

$$
T_{G W}=\max _{s \in \mathcal{S}} \hat{V}^{-1}(s)|\hat{Q}(s)| .
$$

We call this version the MaxK-1 test. Let $m$ be the number of SNPs with $d_{k}=1$. We show the following result in Supplementary Material S3.

Corollary 1. Under conditions given in Theorem 1, if $\hat{\mu}-\mu=o_{p}\left(m^{-1 / 2}\right)$, and $\hat{\omega}^{2}-\omega^{2}=o_{p}(1)$, then $T$ and $T_{G W}$ share the same asymptotic distribution.

According to Corollary 1, as long as we have reasonably precise estimates of $\mu$ and $\omega^{2}$, we can apply the formula given by Theorem 2 to approximate the $p$-value of the MaxK-1 test. Since we can use most of the SNPs to estimate $\mu$ and $\omega^{2}$ according to (6) and (7) under the genome-wide InSIDE assumption, we have $|\Omega|=O_{p}(p)$. Therefore, conditions listed in Corollary 1 are clearly met.

Next, we study the consistency of the MaxK test assuming that $\mu$ and $\omega^{2}$ are known. For the purpose of illustration, we consider the following simplified model. Suppose among the $p$ considered SNPs there are $m=$ $p^{1-\kappa}$ SNPs associated with $X$, with $\beta_{X k} / \sigma_{X k}=\sqrt{2 r \log p}$, where $\kappa$ is the parameter controlling the proportion of SNPs that are associated with the risk factor, and $r$ can be thought as the instrument strength, specifying the SNP's effect size on the risk factor. We further assume $\sigma_{X k}^{2}=\sigma_{Y k}^{2}=\sigma^{2}$ for $k=1, \ldots, p$. Then, under an alternative hypothesis $H_{1}: \theta \neq 0$, the mean 
of $Q(s)$ can be calculated as

$$
\begin{aligned}
& E_{\theta}(S) \equiv E_{\theta}\{Q(s)\} \\
= & \frac{\theta p^{1-\kappa} \sigma \sqrt{2 r \log p}}{\sqrt{\sigma^{2}+\omega^{2}}} E\left\{\frac{\hat{\beta}_{X k}}{\sigma_{X k}} I\left(\frac{\hat{\beta}_{X k}^{2}}{\sigma_{X k}^{2}} \geq 2 s \log p\right)\right\}=\frac{\theta p^{1-\kappa} \sigma \sqrt{2 r \log p}}{\sqrt{\sigma^{2}+\omega^{2}}} h_{s}(r)
\end{aligned}
$$

with

$$
\begin{array}{r}
h_{s}(r)=\sqrt{2 s \log p}\{\bar{\Phi}(\sqrt{2 s \log p}+\sqrt{2 r \log p})+\bar{\Phi}(\sqrt{2 s \log p}-\sqrt{2 r \log p})\} \\
+\phi(\sqrt{2 s \log p}-\sqrt{2 r \log p})-\phi(\sqrt{2 s \log p}+\sqrt{2 r \log p}) .
\end{array}
$$

The variance of $Q(s)$ can be calculated as

$$
\begin{aligned}
& V_{\theta}^{2}(s) \equiv \operatorname{Var}_{\theta}\{Q(s)\} \\
= & V^{2}(s)+\frac{2 \theta^{2} p^{1-\kappa} \sigma^{2} r \log p}{\sigma^{2}+\omega^{2}} \operatorname{Var}\left\{\frac{\hat{\beta}_{X k}}{\sigma_{X k}} I\left(\frac{\hat{\beta}_{X k}^{2}}{\sigma_{X k}^{2}} \geq 2 s \log p\right)\right\} \\
= & V^{2}(s)+\frac{2 \theta^{2} p^{1-\kappa} \sigma^{2} r \log p}{\sigma^{2}+\omega^{2}}\left\{g_{s}(r)-h_{s}(r)\right\},
\end{aligned}
$$

with

$$
\begin{aligned}
g_{s}(r)=(1+2 r \log p) & \{\bar{\Phi}(\sqrt{2 s \log p}+\sqrt{2 r \log p})+\bar{\Phi}(\sqrt{2 s \log p}-\sqrt{2 r \log p})\} \\
+ & (\sqrt{2 s \log p}+\sqrt{2 r \log p}) \phi(\sqrt{2 s \log p}-\sqrt{2 r \log p}) \\
+ & (\sqrt{2 s \log p}-\sqrt{2 r \log p}) \phi(\sqrt{2 s \log p}+\sqrt{2 r \log p})
\end{aligned}
$$

and $V^{2}(s)$ given by $(3)$.

Similar to the proof of Theorem 1, we can establish the asymptotic normality of $Q(s)$ under $H_{1}$ as $V_{\theta}^{-1}(s)\left\{Q(s)-E_{\theta}(s)\right\} \stackrel{D}{\rightarrow} N(0,1)$. Furthermore, we have the following result on the consistency of the MaxK test as $p \rightarrow \infty$.

Theorem 3. Under the above considered model and $H_{1}: \theta \neq 0$, (i) if $r>\rho^{*}(\kappa)$, the power of the MaxK test converges to 1 with the nominal size $\alpha(p)=O\left\{(\log \log p)^{1 / 2}(\log p)^{-1}\right\}$ as $p \rightarrow \infty$; (ii) if $r<\rho^{*}(\kappa)$, the power of the MaxK test converges to 0 when the nominal size $\alpha(p) \rightarrow 0$ as $p \rightarrow \infty$. 
The definition of $\rho^{*}(\kappa)$ and the proof of Theorem 3 are given in Supplementary Material S4. According to this result, given the proportion of $X$ associated SNPs, the MaxK test is consistent if the instrument strength $(r)$ of those SNPs is stronger than $\rho^{*}(\kappa)$. Since $\rho^{*}(\kappa)$ is a monotone increasing function of $\kappa$, the instrument strength that is required to ensure the test consistency becomes higher as the proportion of risk factor associated SNPs decreases.

\subsection{Further relaxation of the genome-wide InSIDE assumption}

Because of the wide spread of SNPs with pleiotropic effects, it can be argued that if a SNP has an effect on $X$, it might have a higher chance of affecting $Y$, comparing to a SNP randomly picked from the genome. Thus, the genome-wide InSIDE assumption might be not be appropriate. We can relax this assumption to allow it to hold conditioning on whether the SNP is associated with the risk factor.

Conditional InSIDE Assumption. For $k=1, \ldots, p, \beta_{Y k}=\theta \beta_{X k}+$ $\alpha_{k}$. $\beta_{X k}$ and $\alpha_{k}$ are conditionally independent given $d_{k}$. Furthermore, $\alpha_{k}$ are i.i.d. with $\alpha_{k} \sim \alpha^{(1)}$ among SNPs having $d_{k}=1$, and $\alpha_{k}$ are i.i.d. with $\alpha_{k} \sim \alpha^{(2)}$ among SNPs having $d_{k}=2$.

The conditional InSIDE assumption is the same as the standard InSIDE assumption on the set of SNPs that are associated with $X$. For SNPs not associated with $X$ (i.e., $d_{k}=2$ ), the requirement of $\alpha_{k} \perp \beta_{X k}$ is always met as $\beta_{X k}$ is constant zero. In that sense, we can regard the conditional InSIDE assumption the same as the standard InSIDE assumption. Both assumptions are reasonable if considered SNPs are not related to any genetic pathway or confounder that affects both $X$ and $Y$.

In the above assumption, $\alpha^{(1)}$, the random effect on $Y$ from a SNP associated with $X$, is allowed to have a different distribution from $\alpha^{(2)}$, which is the random effect from a SNP not associated with $X$. Distributions of $\alpha^{(1)}$ and $\alpha^{(2)}$ can be arbitrary. When the two have the same distribution, the conditional InSIDE assumption reduces to the genome-wide InSIDE 
assumption. To illustrate the difference between the two, we consider the following four-component mixture model suggested by Qi and Chatterjee (2021),

$$
\begin{aligned}
\left(\begin{array}{c}
\beta_{X k} \\
\alpha_{k}
\end{array}\right) \sim \pi_{1}\left(\begin{array}{c}
N\left(0, \sigma_{X}^{2}\right) \\
\delta
\end{array}\right) & +\pi_{2}\left(\begin{array}{c}
N\left(0, \sigma_{X}^{2}\right) \\
N\left(\mu_{Y}, \sigma_{Y}^{2}\right)
\end{array}\right) \\
& +\pi_{3}\left(\begin{array}{c}
\delta \\
N\left(\mu_{Y}, \sigma_{Y}^{2}\right)
\end{array}\right)+\pi_{4}\left(\begin{array}{l}
\delta \\
\delta
\end{array}\right)
\end{aligned}
$$

Under this model, we know $\alpha^{(1)} \sim \frac{\pi_{2}}{\pi_{1}+\pi_{2}} N\left(\mu_{Y}, \sigma_{Y}^{2}\right)+\frac{\pi_{1}}{\pi_{1}+\pi_{2}} \delta$, and $\alpha^{(2)} \sim$ $\frac{\pi_{3}}{\pi_{3}+\pi_{4}} N\left(\mu_{Y}, \sigma_{Y}^{2}\right)+\frac{\pi_{4}}{\pi_{3}+\pi_{4}} \delta$. This model clearly satisfies the conditional InSIDE assumption. It satisfies the genome-wide InSIDE condition if $\pi_{1} \pi_{3}=$ $\pi_{2} \pi_{4}$.

Let the mean and the variance of the random effect $\alpha^{(i)}$ be $\mu_{i}$ and $\omega_{i}^{2}$, $i=1,2$. To adopt the MaxK test under the conditional InSIDE assumption, we can modify the definition of $Q(s)$ as the following,

$$
Q^{*}(s)=\sum_{k=1}^{p}\left\{\frac{\left(\hat{\beta}_{Y k}-\mu_{d_{k}}\right)}{\sqrt{\omega_{d_{k}}^{2}+\sigma_{Y k}^{2}}} \frac{\hat{\beta}_{X k}}{\sigma_{X k}}\right\} I\left(\frac{\hat{\beta}_{X k}^{2}}{\sigma_{X k}^{2}} \geq 2 s \log p\right),
$$

In $Q^{*}(s)$, each SNPs contribution is adjusted by either $\left(\mu_{1}, \omega_{1}^{2}\right)$ or $\left(\mu_{2}, \omega_{2}^{2}\right)$ depending on $d_{k}=1$, or 2 . While in $Q(s)$, the same $\left(\mu, \omega^{2}\right)$ is applied to all SNPs. If we know $d_{k}$ for each SNP, we can estimate $\left(\mu_{1}, \omega_{1}^{2}\right)$ using summary data on SNPs belonging to $\mathcal{M}=\left\{k: d_{k}=1\right\}$ as:

$$
\hat{\omega}_{1}^{2}=\frac{1}{|\mathcal{M}|} \sum_{k \in \mathcal{M}}\left\{\left(\hat{\beta}_{Y k}-\hat{\mu}_{1}\right)^{2}-\hat{\theta}^{2} \hat{\sigma}_{X k}^{2}-\sigma_{Y k}^{2}\right\},
$$

where $\left(\hat{\mu}_{1}, \hat{\theta}\right)$ are estimated coefficients from the linear regression model $\hat{\beta}_{Y k}=\mu_{1}+\theta \hat{\beta}_{X k}+\epsilon_{k}, k \in \mathcal{M}$, with $\epsilon_{k}$ being the error term. The same procedure can be used to estimate $\left(\mu_{2}, \omega_{2}^{2}\right)$ using SNPs in $M \overline{\mathcal{M}}=\left\{k: d_{k}=\right.$ $2\}$. As we do not know $d_{k}$ in real applications, we propose the following strategy to calculate $Q^{*}(s)$. 
Since a SNP in $\mathcal{M}$ tends to have a larger $\left|z_{X k}\right|$ than a SNP from $\mathrm{M} \overline{\mathcal{M}}$, the order of $\left|z_{X k}\right|$ is related to the membership of $\mathcal{M}$. Also, when all independent SNPs from GWAS are considered, it is reasonable to assume $|\overline{\mathcal{M}}|$ is much larger than $|\mathcal{M}|$. Given these two observations, we use the following strategy. First, we arrange all SNPs according to $\left|z_{X k}\right|$ in their descending order. Then, we divide the $p$ SNPs evenly into $L$ groups, so that each group consists of about $\lceil p / L\rceil$ consecutive SNPs. In the following numerical experiments and real data application, we let $\lceil p / L\rceil=100$. Let $G_{l}$ be the set of SNPs belonging to group $l, 1 \leq l \leq L$. For all SNPs in $G_{l}$ we can assume their random effects on $Y$ share a common distribution and estimate its mean and variance as $\hat{\mu}_{l}$ and $\hat{\omega}_{l}^{2}$ according to (10) using summary data on SNPs in $G_{l}$. Finally, we can define the following statistic to approximate $Q^{*}(s)$,

$$
\hat{Q}^{*}(s)=\sum_{l=1}^{L} \sum_{k \in G_{l}}\left\{\frac{\left(\hat{\beta}_{Y k}-\hat{\mu}_{l}\right)}{\sqrt{\hat{\omega}_{l}^{2}+\sigma_{Y k}^{2}}} \frac{\hat{\beta}_{X k}}{\sigma_{X k}}\right\} I\left(\frac{\hat{\beta}_{X k}^{2}}{\sigma_{X k}^{2}} \geq 2 s \log p\right) .
$$

Noticing that If $G_{l} \subseteq \mathcal{M}, \hat{\mu}_{l}$ and $\hat{\omega}_{l}^{2}$ should be valid estimates of $\mu_{1}$ and $\omega_{1}^{2}$. As a result, for SNPs in $G_{l}$, their contributions to $\hat{Q}^{*}(s)$ are approximately the same as ones to $Q^{*}(s)$. The same is true for $G_{l} \subseteq \overline{\mathcal{M}}$. The variance of $\hat{Q}^{*}(s)$ can still be estimated with (4). We define the new MaxK test statistic as

$$
T_{\text {Cond }}=\max _{s \in \mathcal{S}} \hat{V}^{-1}(s)\left|\hat{Q}^{*}(s)\right| \text {. }
$$

We call this version as MaxK-2 test and prove the following result in Supplementary Material S5.

Corollary 2. Given two-sample independent summary data, under the conditional InSIDE assumption, if $G_{l} \subseteq \mathcal{M}$ or $G_{l} \subseteq \overline{\mathcal{M}},\left|G_{l}\right| \rightarrow \infty$ as $p \rightarrow \infty$, for $1 \leq l \leq L$, and if $\beta_{X k} / \sigma_{X k}, 1 \leq k \leq p$, follow a symmetrical distribution around 0 , then under $H_{0}$ we have

$$
\lim _{x \rightarrow+\infty} \frac{1}{x \phi(x)} \operatorname{Pr}\left(T_{\text {Cond }}>x\right)-2 \tau=0,
$$


where $\tau=2^{-1} \log \left\{\hat{V}^{2}\left(s_{a}\right) / \hat{V}^{2}\left(s_{b}\right)\right\}$.

In real applications, we cannot ensure all $G_{l}, 1 \leq l \leq L$, are subsets of $\mathcal{M}$ or $\overline{\mathcal{M}}$. However, when $L$ is relatively large (e.g., $L>100$ ), because of the way all groups are formed and the fact that $|\overline{\mathcal{M}}|$ is much larger than $|\mathcal{M}|, G_{l} \subseteq \mathcal{M}$ or $G_{l} \subseteq \overline{\mathcal{M}}$ should be true (or almost true) for most of $L$ groups, with only a few groups having a mixed bag of SNPs from $\mathcal{M}$ and $\overline{\mathcal{M}}$. Therefore, we still can use Corollary 2 to approximate the $p$-value of the MaxK-2 test.

Corollary 2 requires $\beta_{X k} / \sigma_{X k}$ to be symmetric around zero. Similar condition is also needed by the procedure of Zhao et al. (2019). Since the sign of $\beta_{X k}$ depend on the genotype coding at the SNP, we can adopt a coding scheme to ensure this. We first rearrange SNPs according to $\left|z_{X k}\right|$ in descending order, and then choose the genotype coding in such a way that the sign of $\hat{\beta}_{X k}$ is alternated along the ordered sequence. Given the fact that we usually deal with a large number of SNPs (e.g., $p>50,000$ ), this coding scheme should ensure $\beta_{X k} / \sigma_{X k}$ to be nearly symmetric. Numerical experiments described later confirm that $p$-values can be accurately estimated by the formula given by Corollary 2 with this strategy.

Corollary 2 can be valid under other conditions. For example, if we can assume $\mu_{i}=0, i=1,2$, we can set $\hat{\mu}_{l}=0$ in the calculation of $T_{C o n d}$. Under this situation we can show that the test based on $T_{C o n d}$ is still valid regardless of the distributional property of $\beta_{X k} / \sigma_{X k}$.

\subsection{MaxK test with weakly dependent SNPs}

So far we have described MR methods using summary data from independent SNPs. We will show how to ensure this independency in the Real Data Application Section by removing dependent SNPs. Next we consider the MaxK test with a set of weakly dependent SNPs.

We rearrange SNPs according to their locations on each chromosome. Let $Z_{k}, k=1, \ldots, p$, be the vector of genotypes on the $k^{\text {th }}$ SNP from the two GWAS. Instead of requiring that they are independent, we can allow 
$\mathbf{Z}=\left\{Z_{k}\right\}_{k=1}^{p}$ to have a weak correlation structure called $\rho$-mixing such that their $\rho$-mixing coefficients $\rho_{Z}(k) \leq C v^{k}, k=1, \ldots, p-1$, where $v$ and $C$ are constant values satisfying $0<v<1$, and $C>0$. The $\rho$-mixing coefficient is defined as

$$
\rho_{Z}(k)=\sup _{1 \leq l \leq p-1, \xi \in L^{2}\left(\mathcal{F}_{1}^{l}\right), \eta \in L^{2}\left(\mathcal{F}_{l+k}^{p}\right)}|\operatorname{Corr}(\xi, \eta)|
$$

with $\mathcal{F}_{m}^{n}$ being the $\sigma$-algebra generated by $\left\{Z_{k}\right\}_{k=m}^{n}$. More discussions on the concept of $\rho$-mixing can be found in Doukhan (1994). The $\rho$ mixing dependent structure implies that genotype correlation between two SNP decreases exponentially over their distance, which in general makes sense in human genome, especially after highly correlated SNPs are pruned away. An example of this structure is the autocorrelation structure with the correlation coefficient of $Z_{i}$ and $Z_{j}$ being $\rho_{0}^{|i-j|}$, for some constant value $0<\rho_{0}<1$.

We can show that all results obtained with independent SNPs so far still hold with $\rho$-mixing dependent SNPs. For example, In Supplementary Material S6 we prove the following conclusion.

Theorem 4. For summary data from two separate GWAS on SNPs with $\rho$-mixing dependent structure, under the genome-wide InSIDE assumption, results stated in Theorems 1 and 2 still hold on SNPs with a $\rho$-mixing correlation structure.

\section{Simulation study}

\subsection{Under InSIDE assumption}

We conducted simulation studies to evaluate the performance of the two MaxK tests. We adopted a similar simulation model setup described in Qi and Chatterjee (2021). We assumed that summary data on a set of $p=200,000$ independent SNPs were generated from a risk factor GWAS and an outcome GWAS, where each GWAS had $N$ subjects. Using the same notation as before, we assumed $\beta_{X k}$ be the $k^{\text {th }}$ SNPs effect on $X$, and 
$\alpha_{k}$ be its random effect on $Y$. They followed the four-component mixture model given by (9). The $k^{\text {th }}$ SNPs true marginal effect on $Y$ was defined as $\beta_{Y k}=\theta \beta_{X k}+\alpha_{k}$. We considered two scenarios based on model (9), with Scenario I under the genome-wide InSIDE assumption, and Scenario II under the conditional InSIDE assumption. In both scenarios, we fixed $\sigma_{X}^{2}=\sigma_{Y}^{2}=10^{-5}$. In Scenario I, we set $\pi_{1}=1.96 \%, \pi_{2}=0.04 \%, \pi_{3}=$ $1.96 \%$, and $\pi_{4}=96.04 \%$. Since $\pi_{1} \pi_{3}=\pi_{2} \pi_{4}$ under this setting, $\beta_{X k}$ and $\alpha_{k}$ were generated independently. We further assumed $\mu_{Y}=0$, or 0.005 in (9), which corresponded to the balanced pleiotropy or unbalanced pleiotropy setting. For Scenario II, we set $\pi_{1}=1 \%, \pi_{2}=1 \%, \pi_{3}=1 \%$, and $\pi_{4}=97 \%$. Under this setting, $50 \%$ of SNPs that were associated with $X$ had pleiotropic effects on $Y$. Similar to Scenario I, we chose $\mu_{Y}=0$, or 0.005 to generate balanced or unbalanced pleiotropic effect. For each given mixture model (9), we chose the causal effect $\theta$ within the interval of $[0,0.1]$, and the sample size for each GWAS $(N)$ falling in the range between 300,000 and 500,000.

Given causal effect $\theta$, parameters in model (9), and the sample size $N$, we generated $\left(\beta_{Y k}, \beta_{X k}\right)$ for each SNP independently, and then simulated summary data as $\hat{\beta}_{Y k} \sim N\left(\beta_{Y k}, \frac{1}{N}\right)$, and $\hat{\beta}_{X k} \sim N\left(\beta_{X k}, \frac{1}{N}\right), k=1, \ldots, p$. We replicated the above steps to create 2,000 summary data under each setting for evaluating performances of considered tests, which included the inverse-variance weighted method with multiplicative random effects (IVW) (Burgess et al. 2013), weighted median estimate (W-Median) (Bowden et al., 2016), weighted mode estimate (W-Mode) (Hartwig et al., 2017), MR-Egger (Bowden et al., 2015), MR-Robust (Burgess et al., 2016), contamination mixture (Con-mix) (Burgess et al., 2020), MRMix (Qi and Chatterjee, 2019), as well as two versions of the MaxK test, which were given by (8) and (11). Except the two MaxK tests, all other tests used summary statistics on genome-wide significant SNPs (i.e., SNPs having $X$ association $p$-value less than $\left.5 \times 10^{-8}\right)$. Clearly, more significant SNPs would become available as $N$ increases. In our considered settings, as $N$ increases from $300 \mathrm{k}$ to $500 \mathrm{k}$, the average number of significant SNPs changes from 26 to 
104.

First, we compare all tests under the genome-wide InSIDE Assumption (Scenario I). Table 1 summarizes empirical type I errors for all considered tests when $\mu_{Y}=0$ (i.e., balanced pleiotropy). It is evident from Table 1 that five tests, including MaxK-1, MaxK-2, IVW, MR-Egger, and MRRobust, can maintain their type I errors properly. W-Median, W-Mode, and MRMix are over conservative, especially W-Mode. The Con-mix test has an inflated type I error. Similar conclusions on the type I error evaluation can be reached under unbalanced pleiotropy (Supplementary Table 1). Figure 1 shows the power comparison under balanced pleiotropy with $N=$ $300 \mathrm{k}, 400 \mathrm{k}, 450 \mathrm{k}$, and 500k. We did not consider Con-Mix in the power comparison as it had an inflated type I error rate. From Figure 1 we can see that both versions of the MaxK test have a clear power advantage over other tests. MaxK-1 and MaxK-2 are almost indistinguishable, especially when $N \geq 400 \mathrm{k}$. This suggests that the use of locally estimated mean and variance of the random effect in MaxK-2 does not lead to any noticeable loss of efficiency. Similar conclusions can be made on the power comparison in simulations under unbalanced pleiotropy (Supplementary Figure 1).

Next we compare all tests under the conditional InSIDE assumption (Scenario II). Table 2 and Supplementary Table 2 provide empirical type I error rates under balanced and unbalanced pleiotropy, respectively. We can see from both tables that MaxK-2, IVW, MR-Egger, and MR-Robust can properly maintain their type I errors under all considered sample sizes. The performance of MRMix depends on the number of significant SNPs. It can maintain its type I error appropriately only with a relatively large number of genome-wide significant SNPs. The other tests (W-Median, WMode, Con-mix, and MaxK-1) are either too conversative or liberal. The MaxK-1 test cannot control its type I error as it estimates the mean and variance of the random effect under the Independent Assumption, which is not valid under Scenario II. We excluded Con-mix and MaxK-1 in the power comparison because of their highly inflated type I errors. Figure 2 
and Supplementary Figure 2 show the power comparison under balanced and unbalanced pleiotropy with various sample sizes. According to both figures MaxK-2 appears to be the clear winner.

Finally, to demonstrate the advantage of the proposed MaxK-2 procedure over the testing procedure based on the original K-statistic, we compared its power with tests based on $\hat{V}^{-1}(s)\left|\hat{Q}^{*}(s)\right|$, with a fixed $s$ threshold. We considered $s=0, s=1.64 /(2 \log p)$, and $s=1.96 /(2 \log p)$, and denoted corresponding tests as $\mathrm{K}(0.0), \mathrm{K}(1.64)$ and $\mathrm{K}(1.96)$, respectively. $\mathrm{K}(0.0)$ is equvalent to the orginal K-statistic. We found that the MaxK-2 procedure had a noticeable power advantage over those tests with a fixed threshold. For example, using the same simulation setup under the conditional InSIDE assumption, we compared their powers under various sample sizes and causal effect sizes, with Supplementary Figures 3 and 4 for balanced and unbalanced pleiotropic effects, respectively. Both figures illustrate the robust performance of the MaxK-2 procedure.

\subsection{Under correlated pleiotropic effects}

We evaluated the robustness of MaxK-2 in situations when the InSIDE assumption is not met (i.e., with correlated pleiotropic effects). We considered the InSIDE assumption violated pleiotropy model described in Qi and Chatterjee (2021), where some SNPs have correlated effects (with $10 \%$ correlation coefficient) on the outcome and the risk factor due to their collections with a common mediation factor. We found that MaxK-2 can control its type I error reasonably well when less than $10 \%$ of risk factor associated SNPs have correlated effect on the outcome (Supplementary Table 3). But it tends to have inflated type I error when the percentage of SNPs with correlated effect becomes large (Supplementary Table 3). This is expected as MaxK-2 is derived under the Conditional InSIDE assumption.

\section{Real application}

Jones et al. (2019) recently studied the genetic basis underlying various human sleep behaviors. In their study, they conducted MR analyses to iden- 
tify risk factors with causal effects on sleep behaviors. In particular, they considered eight sleep behaviors quantified by accelerometer-derived measures and used MR to assess whether waist-hip-ratio (WHR) can causally affect them. Here we applied our new test to re-assess those relationships. The summary data on SNPs association with WHR (after the adjustment of BMI) were obtained from Shungin et al. (2015). Summary data on SNPs association with each sleep behavior was from Jones et al. (2019). The eight considered sleep behaviors (inverse-normalized) are listed in Table 3.

We preprocessed the summary data with the following criteria. We restricted SNPs to those sharing between WHR and sleep behavior GWAS and with minor allele frequencies (MAFs) larger than 2\%. MAFs were estimated with European reference genomes from the 1000 Genomes (Genomes et al., 2015). Then we used the clumping function of PLINK (Purcell et al. 2007) with $r^{2}=0.1$ within a window size of $1000 \mathrm{~kb}$ as the linkage disequilibrium threshold to select a set of independent SNPs. When we applied the clumping procedure, we randomly picked index SNPs without referring to their levels of association with WHR and sleep behaviors, in order to ensure that there was no selection bias. In the end we had 95,819 SNPs for the MaxK test. For MR analyses with other considered procedures, we used a set of 56 independent SNPs among the ones that were genome-wide significantly associated with WHR (i.e., with $p$-value less than $5 \times 10^{-8}$ ) in the WHR GWAS.

Results are summarized in Table 3. We did not present results from Con-mix and MaxK-1 as they had inflated type I errors according to our simulation results. From Table 3 we can see that the MaxK-2 test can detect most signals among all considered tests, with five out of eight outcomes having their MaxK-2 test $p$-values less than 0.05, and three having their MaxK-2 test p-values less than the Bonferroni threshold (i.e., 0.05/8 $\approx$ 0.006). Among all considered tests, the MaxK-2 test has the most significant result on four outcomes (i.e., L5 timing, Sleep duration variability, Sleep efficiency, and Sleep midpoint timing), while MRMix and MR-robust each 
has one.

In this real data example, except the proposed MaxK test, the other seven tests used summary data on 56 SNPs that were genome-wide significantly associated with WHR. As we have pointed out before, the requirement of using only genome-wide significant SNPs is the major limitation for those approaches. By combining signals throughout the genome, instead of relying on a few SNPs, the Max-K test apparently can detect the casual effect of WHR on more sleep habits.

\section{Discussion}

We propose a new MR test (called the MaxK test) that can take full advantage of information generated from genome-wide association studies. Unlike most existing procedures that rely on a few SNPs that demonstrate strong evidence for their association with the risk factor, the MaxK test synthesizes evidence of causal effect from tens of thousands of SNPs studied by GWAS. This test can properly control its type I error under the InSIDE assumption with balanced or unbalanced pleiotropy. It is more powerful than existing approaches even when there is only a small proportion (e.g., 1 or $2 \%$ ) of SNPs carrying the signal.

It has been challenging to develop MR procedures with properly controlled type I error when some IVs have correlated pleiotropic effects. The proposed method has difficulty maintaining its type I error when a large proportion of considered SNPs have correlated pleiotropic effects. Another limitation is that our method does not provide an estimate of the causal effect. Given its promising performance under the InSIDE assumption, it would be worthwhile to further improve the MaxK procedure so it can have a more robust performance when SNPs with correlated pleiotropic effects are used as IVs. Finally, the proposed procedure focuses on testing the null hypothesis that the risk factor has no casual effect on the outcome. It does not provide an estimate of the causal effect. Further investigations are needed to expand the procedure for evaluating the magnitude of the casual effect. 


\section{Supplementary Material}

All technical details and additional numeric results are relegated to the online Supplementary Material.

\section{Acknowledgement}

The study utilized the computational resource of the NIH Biowulf cluster (https://hpc.nih.gov/). The research of Dr. Lu Deng was partially supported by the National Natural Science Foundation of China grant \#12101331. The authors would like to thank the Associate Editor and two Referees whose insightful comments lead to an improved manuscript.

\section{References}

Bowden, J., Davey Smith, G., and Burgess, S. (2015). Mendelian randomization with invalid instruments: effect estimation and bias detection through Egger regression. Int J Epidemiol 44, 512-525.

Bowden, J., Davey Smith, G., Haycock, P. C., and Burgess, S. (2016). Consistent estimation in Mendelian randomization with some invalid instruments using a weighted median estimator. Genet Epidemiol 40, 304-314.

Bowden, J., Del Greco, M. F., Minelli, C., Davey Smith, G., Sheehan, N., and Thompson, J. (2017). A framework for the investigation of pleiotropy in two-sample summary data Mendelian randomization. Stat Med 36, 1783-1802.

Burgess, S., Bowden, J., Dudbridge, F., and Thompson, S. G. (2016). Robust instrumental variable methods using multiple candidate instruments with application to Mendelian randomization. arXiv 1606.03279.

Burgess, S., Butterworth, A., and Thompson, S. G. (2013). Mendelian randomization analysis with multiple genetic variants using summarized data. Genet Epidemiol 37, 658-665.

Burgess, S., Foley, C. N., Allara, E., Staley, J. R., and Howson, J. M. M. (2020). A robust 
and efficient method for Mendelian randomization with hundreds of genetic variants. Nat Commun 11, 376 .

Burgess, S., Scott, R. A., Timpson, N. J., Davey Smith, G., Thompson, S. G., and Consortium, E.-I. (2015). Using published data in Mendelian randomization: a blueprint for efficient identification of causal risk factors. Eur J Epidemiol 30, 543-552.

Deng, L., Zhang, H., and Yu, K. (2020). Power calculation for the general two-sample Mendelian randomization analysis. Genet Epidemiol 44, 290-299.

Didelez, V., and Sheehan, N. (2007). Mendelian randomization as an instrumental variable approach to causal inference. Stat Methods Med Res 16, 309-330.

Donoho, D. L., and Jin, J. (2004). Higher criticism for detecting sparse heterogeneous mixtures. Annals of Statistics 32, 962-994.

Doukhan, P. (1994). Mixing: Properties and Examples. In Lecture Notes in Statistics. New York: Springer.

Evangelou, E., Warren, H. R., Mosen-Ansorena, D., et al. (2018). Genetic analysis of over 1 million people identifies 535 new loci associated with blood pressure traits. Nat Genet 50, 1412-1425.

Genomes Project, C., Auton, A., Brooks, L. D., et al. (2015). A global reference for human genetic variation. Nature 526, 68-74.

Guo, Z., Kang, H., Cai, T. T., and Small, D. S. (2018). Confidence intervals for causal effects with invalid instruments by using two-stage hard thresholding with voting. J $R$ Statist Soc $B$ 80, 793-815.

Hartwig, F. P., Davey Smith, G., and Bowden, J. (2017). Robust inference in summary data Mendelian randomization via the zero modal pleiotropy assumption. Int J Epidemiol 46, 1985-1998.

Hemani, G., Zheng, J., Elsworth, B., et al. (2018). The MR-Base platform supports systematic causal inference across the human phenome. Elife $\mathbf{7}$. 
Jones, S. E., van Hees, V. T., Mazzotti, D. R., et al. (2019). Genetic studies of accelerometerbased sleep measures yield new insights into human sleep behaviour. Nat Commun 10, 1585.

Kang, H., Lee, Y., Cai, T. T., and Small, D. S. (2020). Two robust tools for inference about causal effects with invalid instruments. Biometrics doi: 10.1111/biom.13415. Epub ahead of print. PMID: 33616910.

Kang, H., Zhang, A., Cai, T. T., and Small, D. S. (2016) Instrumental variables estimation with some invalid instruments and its application to mendelian randomization. J Am Stat Assoc 111, 132-144,

Kleibergen, F. (2002). Pivotal statistics for testing structural parameters in instrumental variables regression. Econometrica 70, 1781-1803.

Lawlor, D. A., Harbord, R. M., Sterne, J. A., Timpson, N., and Davey Smith, G. (2008). Mendelian randomization: using genes as instruments for making causal inferences in epidemiology. Stat Med 27, 1133-1163.

Morrison, J., Knoblauch, N,, Marcus, J. H., Stephens, M. and He, X. (2020) Mendelian randomization accounting for correlated and uncorrelated pleiotropic effects using genome-wide summary statistics. Nat Genet 52, 740-747.

Petrov, V. V. (1995). Limit theorems of probability theory. Sequences of independent random variables. New York: Oxford University Press.

Purcell, S., Neale, B., Todd-Brown, K., et al. (2007). PLINK: a tool set for whole-genome association and population-based linkage analyses. Am J Hum Genet 81, 559-575.

Qi, G., and Chatterjee, N. (2019). Mendelian randomization analysis using mixture models for robust and efficient estimation of causal effects. Nat Commun 10, 1941.

Qi, G., and Chatterjee, N. (2019b). A comprehensive evaluation of methods for Mendelian randomization using realistic simulations and an analysis of 38 biomarkers for risk of type- 2 diabetes. Int J Epidemiol 50, 1335-1349.

Shungin, D., Winkler, T. W., Croteau-Chonka, D. C., et al. (2015). New genetic loci link adipose 
and insulin biology to body fat distribution. Nature 518, 187-196.

Siegmund, D. (2002). Upward bias in estimation of genetic effects. Am J Hum Genet 71, 11831188.

Smith, G. D., and Ebrahim, S. (2003). 'Mendelian randomization': can genetic epidemiology contribute to understanding environmental determinants of disease? Int J Epidemiol 32, $1-22$.

Tchetgen, E. J. T., Sun, B., and Walter, S. (2017). The GENIUS approach to robust Mendelian randomization inference. Statist Sci 36, 443 - 464,

Wang, S., and Kang, H. (2019). Weak-Instrument robust tests in two-sample summary-data Mendelian randomization. arXiv 1909.06950

Windmeijer, F., Farbmacher, H., Davies, N., and Davey Smith, G. (2018). On the use of the lasso for instrumental variables estimation with some invalid instruments. J Am Stat Assoc 114, 1339-1350.

Xue, H., Shen, X., and Pan, W. (2021). Constrained maximum likelihood-based Mendelian randomization robust to both correlated and uncorrelated pleiotropic effects. Am J Hum Genet 108, 1251-1269.

Yengo, L., Sidorenko, J., Kemper, K. E., et al. (2018). Meta-analysis of genome-wide association studies for height and body mass index in approximately 700000 individuals of European ancestry. Hum Mol Genet 27, 3641-3649.

Yu, K., Chatterjee, N., Wheeler, W., et al. (2007). Flexible design for following up positive findings. Am J Hum Genet 81, 540-551.

Zhao, Q., Chen, Y., Wang, J., and Small, D. S. (2019). Powerful three-sample genome-wide design and robust statistical inference in summary-data Mendelian randomization. Int $J$ Epidemiol 48, 1478-1492.

Zhao, Q., Wang, J., Hemani, G., Bowden, J., and Small, D. S. (2020). Statistical inference in two-sample summary-data Mendelian randomization using robust adjusted profile score. 
Annals of Statistics 48, 1742-1769.

Zhong, P., Chen, S. X., and Xu, M. (2013). Tests alternative to Higher criticism for high dimensional means under sparsity and column-wise dependence. Annals of Statistics 41, 2820-2851. 
Table 1: Simulation results on type I errors under the genome-wide InSIDE assumption with balanced pleiotropy. Results are summarized based on performances over 2000 datasets generated from two GWAS of equal sample size $(N)$. Each simulated dataset consists of summary statistics on 200,000 independent SNPs.

\begin{tabular}{c|ccccc}
\hline & \multicolumn{5}{|c}{ Sample size $N$ (average number of significant SNPs) } \\
& MR method & \\
& $300 \mathrm{k}(26)$ & $350 \mathrm{k}(41)$ & $400 \mathrm{k}(59)$ & $450 \mathrm{k}(80)$ & $500 \mathrm{k}(104)$ \\
\hline MaxK-1 & 0.044 & 0.048 & 0.046 & 0.048 & 0.049 \\
MaxK-2 & 0.038 & 0.044 & 0.042 & 0.045 & 0.044 \\
IVW & 0.045 & 0.043 & 0.045 & 0.042 & 0.048 \\
W-Median & 0.026 & 0.023 & 0.021 & 0.025 & 0.019 \\
W-Mode & 0.001 & 0.001 & 0.001 & 0.001 & 0.002 \\
IVW-Robust & 0.040 & 0.041 & 0.042 & 0.042 & 0.046 \\
MR-Egger & 0.041 & 0.048 & 0.045 & 0.056 & 0.045 \\
Con-mix & 0.122 & 0.155 & 0.170 & 0.192 & 0.208 \\
MRMix & 0.031 & 0.028 & 0.024 & 0.021 & 0.016 \\
\hline
\end{tabular}

a. Each summary data is generated from two GWAS of equal sample size $N$. The average number of significant SNPs is the number of SNPs with their risk factor association $p$-values less than $5 \times 10^{-8}$, averaged over 2000 simulated datasets.

b. Both MaxK-1 and MaxK-2 use summary statistics on 200,000 independent SNPs. All other tests use summary statistics on SNPs that are genome-wide significantly associated with the risk factor. 
Table 2: Simulation results on type I errors under the conditional InSIDE assumption with balanced pleiotropy. Results are summarized based on performances over 2000 datasets generated from two GWAS of equal sample size $(N)$. Each simulated dataset consists of summary statistics on 200,000 independent SNPs.

\begin{tabular}{c|ccccc}
\hline & \multicolumn{5}{|c}{ Sample size $N$ (average number of significant SNPs) } \\
& MR method & \\
& $300 \mathrm{k}(26)$ & $350 \mathrm{k}(41)$ & $400 \mathrm{k}(59)$ & $450 \mathrm{k}(80)$ & $500 \mathrm{k}(104)$ \\
\hline MaxK-1 & 0.254 & 0.291 & 0.325 & 0.347 & 0.365 \\
MaxK-2 & 0.049 & 0.052 & 0.057 & 0.058 & 0.058 \\
IVW & 0.064 & 0.073 & 0.057 & 0.056 & 0.051 \\
W-Median & 0.081 & 0.079 & 0.076 & 0.073 & 0.071 \\
W-Mode & 0.012 & 0.004 & 0.004 & 0.004 & 0.004 \\
IVW-Robust & 0.061 & 0.064 & 0.062 & 0.055 & 0.060 \\
MR-Egger & 0.058 & 0.053 & 0.048 & 0.048 & 0.058 \\
Con-mix & 0.196 & 0.215 & 0.206 & 0.210 & 0.218 \\
MRMix & 0.089 & 0.069 & 0.059 & 0.058 & 0.052 \\
\hline
\end{tabular}

a. Each summary data is generated from two GWAS of equal sample size $N$. The average number of significant SNPs is the number of SNPs with their risk factor association p-values less than $5 \times 10^{-8}$, averaged over 2000 simulated datasets.

b. Both MaxK-1 and MaxK-2 use summary statistics on 200,000 independent SNPs. All other tests use summary statistics on SNPs that are genome-wide significantly associated with the risk factor. 
Table 3: MR testing results ( $p$-values) on the casual effect of waist-hip-ratio on eight sleep traits.

\begin{tabular}{|c|c|c|c|c|}
\hline \multirow[b]{2}{*}{ Outcome } & \multicolumn{4}{|c|}{ MR method } \\
\hline & Maxk-2 & IVW & W-Median & W-Mode \\
\hline Diurnal inactivity & $9.84 \mathrm{E}-01$ & $1.20 \mathrm{E}-01$ & $9.12 \mathrm{E}-02$ & $6.14 \mathrm{E}-01$ \\
\hline L5 timing & $2.44 \mathrm{E}-02$ & $5.83 \mathrm{E}-01$ & $5.40 \mathrm{E}-01$ & $3.27 \mathrm{E}-01$ \\
\hline M10 timing & $8.18 \mathrm{E}-01$ & $4.76 \mathrm{E}-01$ & $1.64 \mathrm{E}-01$ & $3.04 \mathrm{E}-01$ \\
\hline Num. nocturnal sleep episodes & 4.67E-01 & $9.75 \mathrm{E}-01$ & 3.60E-01 & 5.39E-01 \\
\hline Sleep duration & 1.69E-04 & $7.56 \mathrm{E}-05$ & $1.33 \mathrm{E}-02$ & $5.78 \mathrm{E}-01$ \\
\hline Sleep duration variability & $1.07 \mathrm{e}-03$ & $5.98 \mathrm{E}-02$ & $1.27 \mathrm{E}-02$ & $1.29 \mathrm{E}-01$ \\
\hline Sleep efficiency & $2.26 \mathrm{E}-04$ & $2.40 \mathrm{E}-03$ & $2.85 \mathrm{E}-03$ & $1.51 \mathrm{E}-01$ \\
\hline \multirow[t]{2}{*}{ Sleep midpoint timing } & $3.45 \mathrm{e}-02$ & $1.34 \mathrm{E}-01$ & 4.40E-01 & 7.64E-01 \\
\hline & IVW-Robust & MR-Egger & MRMix & \\
\hline Diurnal inactivity & 7.88E-02 & $2.84 \mathrm{E}-01$ & $2.26 \mathrm{E}-01$ & \\
\hline L5 timing & $9.29 \mathrm{E}-01$ & 8.07E-01 & $5.79 \mathrm{E}-01$ & \\
\hline M10 timing & 4.16E-01 & $2.62 \mathrm{E}-01$ & 3.39E-04 & \\
\hline Num. nocturnal sleep episodes & $6.37 \mathrm{E}-01$ & $2.44 \mathrm{E}-02$ & $8.26 \mathrm{E}-01$ & \\
\hline Sleep duration & $5.53 \mathrm{E}-05$ & $2.25 \mathrm{E}-01$ & 4.67E-01 & \\
\hline Sleep duration variability & $5.32 \mathrm{E}-02$ & $3.45 \mathrm{E}-02$ & $4.31 \mathrm{E}-01$ & \\
\hline Sleep efficiency & $1.01 \mathrm{E}-03$ & $8.50 \mathrm{E}-01$ & 7.30E-01 & \\
\hline Sleep midpoint timing & $9.30 \mathrm{E}-02$ & $6.47 \mathrm{E}-01$ & 7.02E-02 & \\
\hline
\end{tabular}


Figure 1: Simulation results on power comparisons under the genome-wide InSIDE assumption with balanced pleiotropy. Results are summarized based on performances over 2000 simulated datasets under a given causal effect (theta) and sample size $(N)$. Each simulated dataset consists of summary statistics on 200,000 independent SNPs generated from two GWAS of equal sample size $N$. (a) Both GWAS have sample size $N=300 \mathrm{k}$, with an average number of 26 SNPs that are genome-wide significantly associated with the risk factor; (b). Sample size $N=400 \mathrm{k}$, and an average number of 59 significant SNPs; (c). Sample size $N=450 \mathrm{k}$, and an average number of 80 significant SNPs; and (d). Sample size $N=500 \mathrm{k}$, and an average number of 104 significant SNPs.
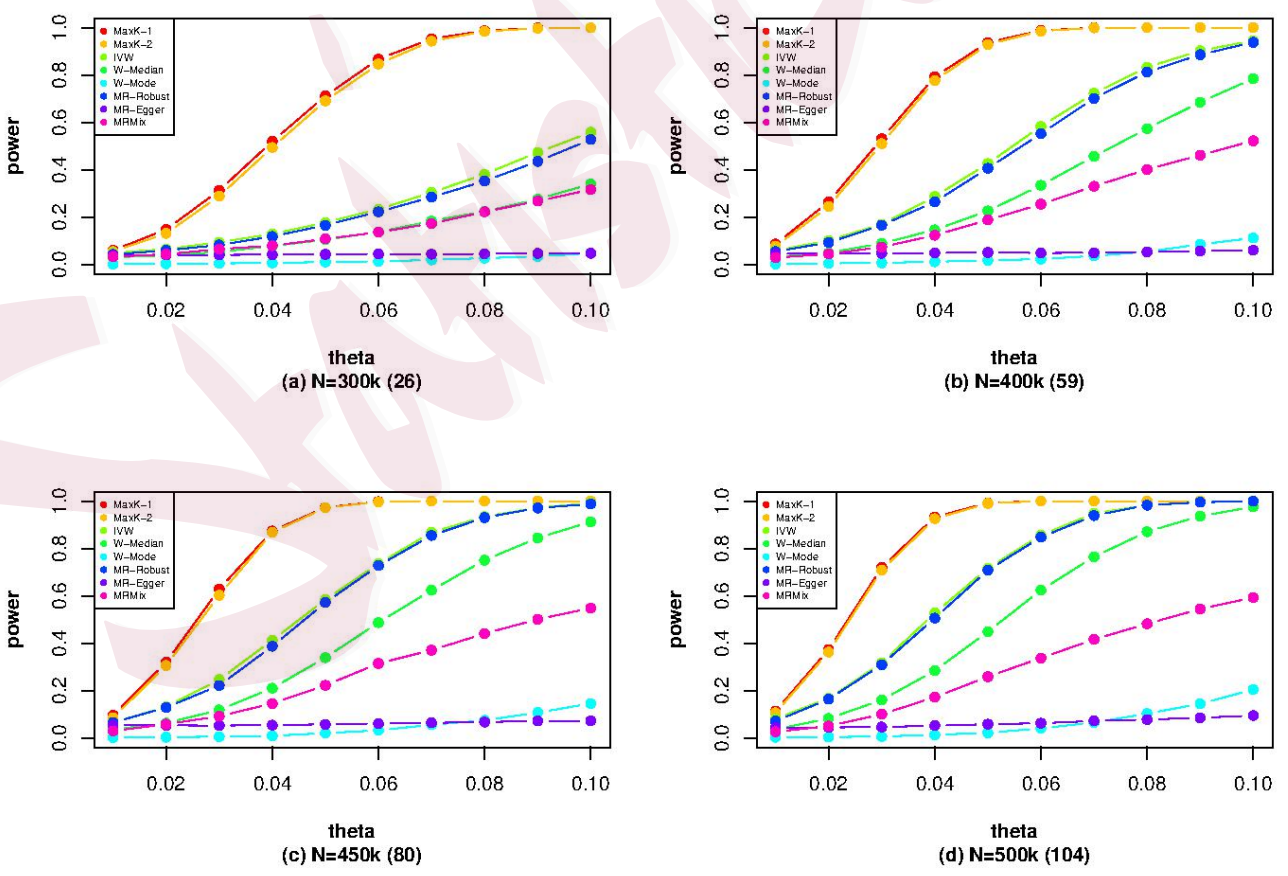
Figure 2: Simulation results on power comparisons under the conditional InSIDE assumption with balanced pleiotropy. Results are summarized based on performances over 2000 simulated datasets under a given causal effect (theta) and sample size $(N)$. Each simulated dataset consists of summary statistics on 200,000 independent SNPs generated from two GWAS of equal sample size $N$. (a) Both GWAS have sample size $N=300$ k, with an average number of 26 SNPs that are genome-wide significantly associated with the risk factor; (b). Sample size $N=400 \mathrm{k}$, and an average number of 59 significant SNPs; (c). Sample size $N=450 \mathrm{k}$, and an average number of 80 significant SNPs; and (d). Sample size $N=500 \mathrm{k}$, and an average number of 104 significant SNPs.
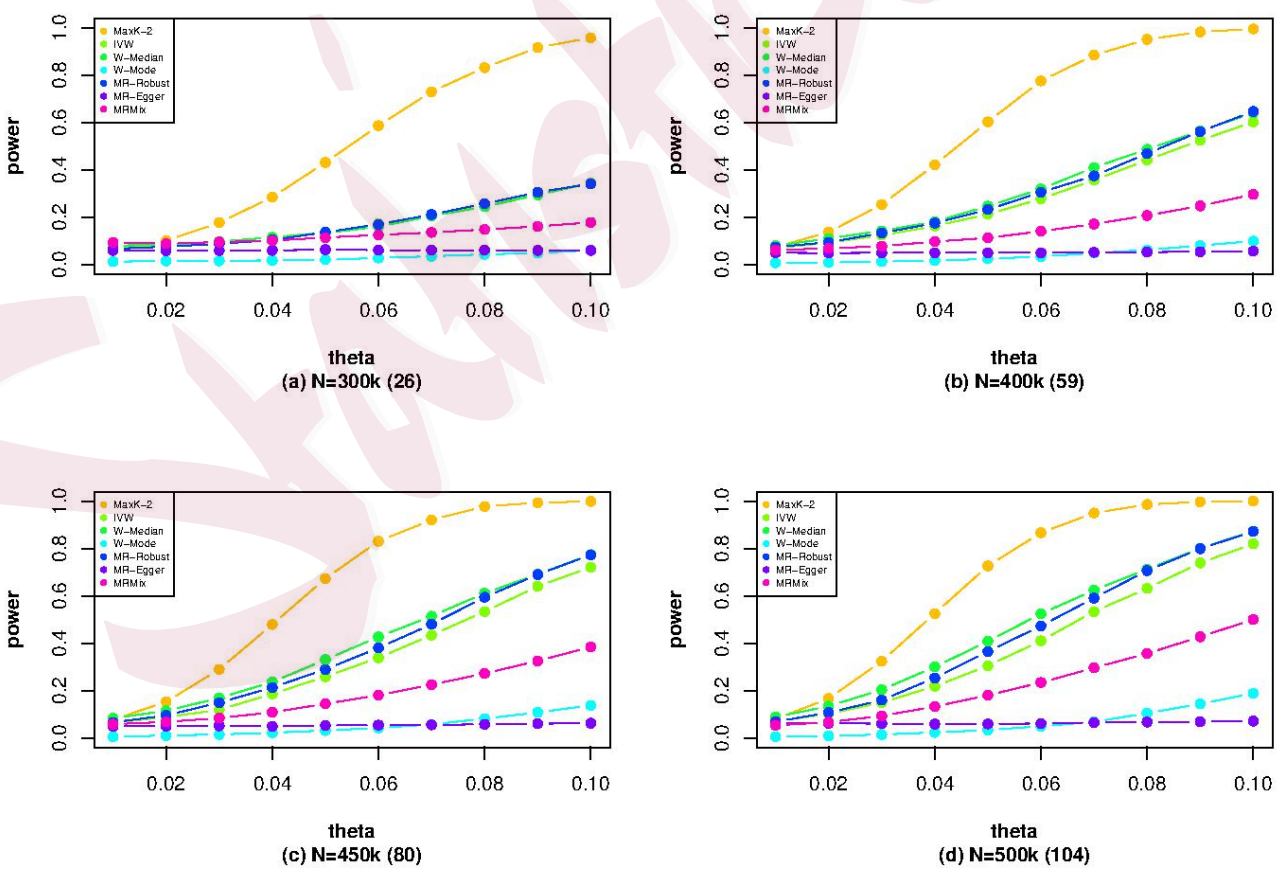